\title{
Simple estimates of excitation energy sharing between heavy and light fragments in heavy-ion reactions
}

\author{
C. H. Dasso \\ The Niels Bohr Institute and NORDITA, Blegdamsvej 17, DK-2100 Copenhagen 0 , Denmark \\ M. Lozano \\ Departamento de Fisica Atomica y Nuclear, Apdo. 1065, Universidad de Sevilla, 41080 Sevilla, Spain \\ G. Pollarolo \\ Istituto di Fisica Teorica dell' Università di Torino and Istituto Nazionale di Fisica Nucleare, \\ Sezione di Torino, Torino, Italy \\ (Received 26 August 1985)
}

\begin{abstract}
Qualitative arguments are used to estimate the ratio of excitation energies between heavy and light fragments for asymmetric heavy-ion collisions. The value of this quantity is linked to the relative role played by inelastic and transfer degrees of freedom and thereby to an approximate function of the total kinetic energy loss. A numerical analysis that confirms the trends anticipated by the simple arguments is performed for the reactions ${ }^{56} \mathrm{Fe}+{ }^{238} \mathrm{U}$ and ${ }^{86} \mathrm{Kr}+{ }^{208} \mathrm{~Pb}$ at bombarding energies in the laboratory of 476 and 1565 $\mathrm{MeV}$, respectively.
\end{abstract}

In many heavy-ion collisions a large fraction of the energy initially available in the relative motion of the nuclei is converted in intrinsic excitation energy of the reacting fragments. The question of the approximate division of this total kinetic energy loss between the fragments has attracted considerable interest from early stages in the study of these phenomena. This was in part motivated by the belief that an understanding of the ratio in which the excitation energy was deposited in the two colliding ions would help to identify the class of mechanisms responsible for the large observed energy (and angular momentum) transfers.

Empirical evidence on this question was provided by measurements of the distributions of neutrons emitted from the highly excited reaction products. Advocates of the statistical pictures (in which a degree of local equilibrium is required) have naturally tended to interpret early experimental results as consistent with a division of excitation energy between the fragments given by the same ratio as that of their masses. ${ }^{1-3}$ This is, in principle, what is required to ascribe equal temperature to projectile and target.

This paper is motivated by the recent appearance in the literature of results based on the same class of experimental data for the reaction ${ }^{56} \mathrm{Fe}+{ }^{238} \mathrm{U}$ at $476 \mathrm{MeV} .{ }^{4}$ These exhibit ratios of the excitation energy between the heavy and light fragments far away from the value 4.2 required by the simple formula $E_{H}^{*} / E_{L}^{*}=A_{H} / A_{L}$. Refinements on the statistical picture associated with mass transfer degrees of freedom have now been advanced to account for these large deviations. An example of this line of thought is given by Moretto and $\mathrm{Lanza}^{5}$ who have proposed a somewhat involved feedback mechanism to reconcile the theory with the experimental evidence. Theoretical results in agreement with the experiment have also been obtained with the dynamical transport model of Randrup.,

It should be emphasized that there is no actual need to patch up the statistical predictions. Indeed, deviations from the thermal equilibrium picture follow naturally as a consequence of excitation of isoscalar density modes. The presence of large deformations in the nuclear shapes has been, on the one hand, quite unambiguously inferred from the experimental data. Moreover, microscopic calculations within the time-dependent Hartree-Fock approximation ${ }^{7}$ and by the surface excitation model of Ref. 8 have exhibited the important role played by these degrees of freedom. In what follows we shall refer to them as inelastic modes albeit they should be considered as generalized ones given the large amount of particle transfer that occurs simultaneously with the deformation of the nuclear volumes. Perhaps it is timely to make a simple estimate of the magnitude of these effects as they do not scale with mass number in the same way as the statistical transfers do.

A key ingredient to address this problem lies on the relative importance of these two mechanisms in the total energy dissipation. Although this question has been widely debated there is no conclusive answer yet. Calculations which take both processes explicitly into account ${ }^{9}$ showed a rather even share in the total energy loss between inelastic and transfer modes. Beyond these simple order-of-magnitude results one expects a transition from a situation in which inelastic processes dominate for small energy loss and for energies not too high over the Coulomb barrier to conditions in which particle transfer takes over for large energy losses and very high bombarding energies. In what follows we denote by $\alpha$ the ratio

$$
\alpha=\frac{E_{\mathrm{transfer}}^{*}}{E^{*}},
$$

where $E^{*}=E_{\text {inelastic }}^{*}+E_{\text {transfer }}^{*}$ is the total excitation energy.

The number and multipolarities of the different degrees of freedom of the nuclear surfaces in both fragments is roughly the same, independent of their size. Thus, one can estimate the ratio between the inelastic excitation of the heavy and light fragments by considering just a given state for either nucleus. The energy accumulated by one such mode $n$ can be written as

$$
E_{\text {inelastic }}^{*}(n)=\hbar \omega_{n}\left|\int_{-\infty}^{\infty} F_{n}(t) e^{i \omega} n^{t} d t\right|^{2},
$$

(c) 1985 The American Physical Society 
where $F_{n}(t)$ stands for the time-dependent function which induces the excitation of the state. Let us focus our attention on the $A$ dependence of the previous expression.

For collective surface modes the function $F_{n}(t)$ is of the form

$$
F_{n}(t)=\sqrt{\hbar \omega_{n} / 2 C_{n}} R \partial \mathrm{U}(r(t)) / \partial r .
$$

Since the trajectory of relative motion and the ion-ion potential $\mathrm{U}(r)$ is common for both reaction partners the $A$ dependence is contained in the square root and radius factors. Leaving aside the $Q$-value effects associated with the exponential factor, the distribution of energy between projectile and target modes is determined by the quantity

$$
q=\frac{\left(\hbar \omega_{n}\right)^{2} R^{2}}{2 C_{n}}
$$

Excitation energies of nuclei scale as $A^{-1 / 3}$ while their radii go as $A^{1 / 3}$. In principle, the $A$ dependence of the restoring force parameter should go as $A^{-2 / 3}$. However, the situation here is not clear-cut as the parameter $C$ depends quite sensitively on the details of the shell structure. It should also be noted that here we have focused our attention on the nuclear part of the interaction coupling but that for lower multipolarities the results will be somewhat affected by the Coulomb forces. We may refer to Figs. 6-28 of Ref. 10 for a survey of the quadrupole restoring force parameter over a very large range of masses. Following the liquid-drop systematics one could then, as a first approximation, take the restoring force parameter to be roughly constant. To allow for some flexibility we thus divide the inelastic excitation energy according to the power law

$$
E_{H / L, \text { inelastic }}^{*}=\frac{A_{H / L}^{p}}{\left(A_{L}^{p}+A_{H}^{p}\right)}(1-\alpha) E^{*},
$$

where $p$ is somewhere in the interval $\left[-\frac{2}{3}, 0\right]$.

Assuming the fraction of the energy loss due to transfer to be distributed in the standard statistical way we find that the ratio of the energies deposited in the heavy and light fragments is then given by

$$
\frac{E_{H}^{*}}{E_{L}^{*}}=\frac{A_{H} /\left(A_{L}+A_{H}\right) \alpha+A_{H}^{p} /\left(A_{L}^{p}+A_{H}^{p}\right)(1-\alpha)}{A_{L} /\left(A_{L}+A_{H}\right) \alpha+A_{L}^{p} /\left(A_{L}^{p}+A_{H}^{p}\right)(1-\alpha)} .
$$

In Fig. 1 we display the values of this quantity as a function of $\alpha$ for the particular choice $A_{H}=238, A_{L}=56$ which corresponds to the case discussed in Ref. 4. Besides the curve for $p=-\frac{1}{3}$ we also show the range covered by the function for values of $p$ in the interval $\left[-1, \frac{1}{3}\right]$ to give an idea how sensitive these estimates are to other $A$ dependences of the restoring force parameter. Ratios $E_{H}^{*} / E_{L}^{*}$ of the order of two easily result from values of $\alpha$ in the range [0.25, 0.75], in consistency with the findings of Ref. 9. Following the considerations mentioned above one can establish a rough correspondence between the values of $\alpha$ and the total kinetic energy loss (TKEL); low and high values of TKEL would then be at the left and right sides of Fig. 1, respectively.

The previous formula is useful for understanding the characteristics of the energy balance and to estimate the expected ratios for any other combination of projectile and target. For the particular reaction under consideration we have tried to substantiate the results of the current analysis by

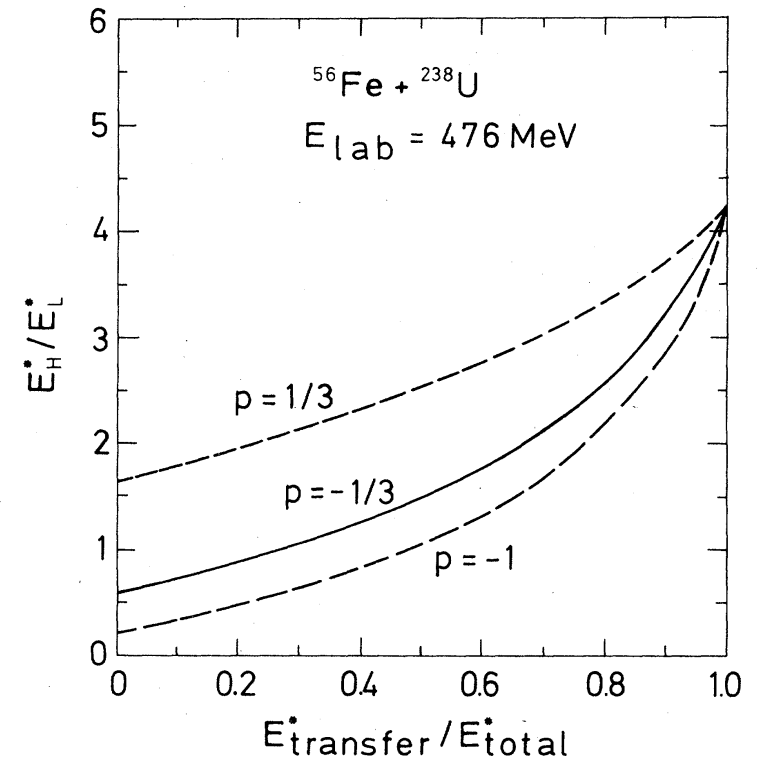

FIG. 1. Ratio of the energies deposited in the heavy and light fragments as a function of $\alpha$ for a range of values of $p$ (see text).

performing a complete calculation within the model of Ref. 8. For this purpose we have used the semiclassical coupled channel code TORINO ${ }^{11}$ with the response functions for each nucleus given in Table I of Ref. 8. The simplest procedure would be to calculate the average trajectories as a function of the impact parameter and then to unfold the ratio of excitation energies as a function of the total kinetic energy loss. However, since the ratio of energies $E_{H}^{*} / E_{L}^{*}$ is not expected to be in one-to-one correspondence with the partial wave number, we have performed the calculations in such a way as to obtain the magnitude of the fluctuations which originate in the coupling to the surface modes (for details cf., e.g., Refs. 8 and 12). In Fig. 2 the results for the reaction ${ }^{56} \mathrm{Fe}+{ }^{238} \mathrm{U}$ at $476 \mathrm{MeV}$ are shown in comparison with the experimental data of Ref. 4. The estimates for the spread around the average results (empty circles) are indicated by dotted lines. We have also performed calculations for the same system at higher bombarding energies which show a gradual increase of the value for the ratio $E_{H}^{*} / E_{L}^{*}$, as expected from the simple arguments illustrated in Fig. 1.

In Fig. 3 we compare the results of a similar calculation for the reaction ${ }^{86} \mathrm{Kr}+{ }^{208} \mathrm{~Pb}$ at $1565 \mathrm{MeV}$ with data extracted from the recent measurements of Sohlbach et al. ${ }^{13}$ Both theoretical and experimental results are in this case closer to the thermal ratio. From the observed value of $E_{H}^{*} / E_{L}^{*}$ one extracts $\alpha \simeq 0.7$ consistent with the higher bombarding energy in this case. We note that in both reactions the estimated size of the fluctuations is larger than the reported experimental errors. It would thus be interesting to have measurements for the second moments of these quantities. Since the expected widths from statistical origins are relatively small, evidence for large fluctuations may confirm the importance of quantal effects in the process.

In summary, we find that qualitative arguments, confirmed by model calculations, provide a simple way to estimate the sharing of excitation energy between the fragments in heavy-ion collisions. 


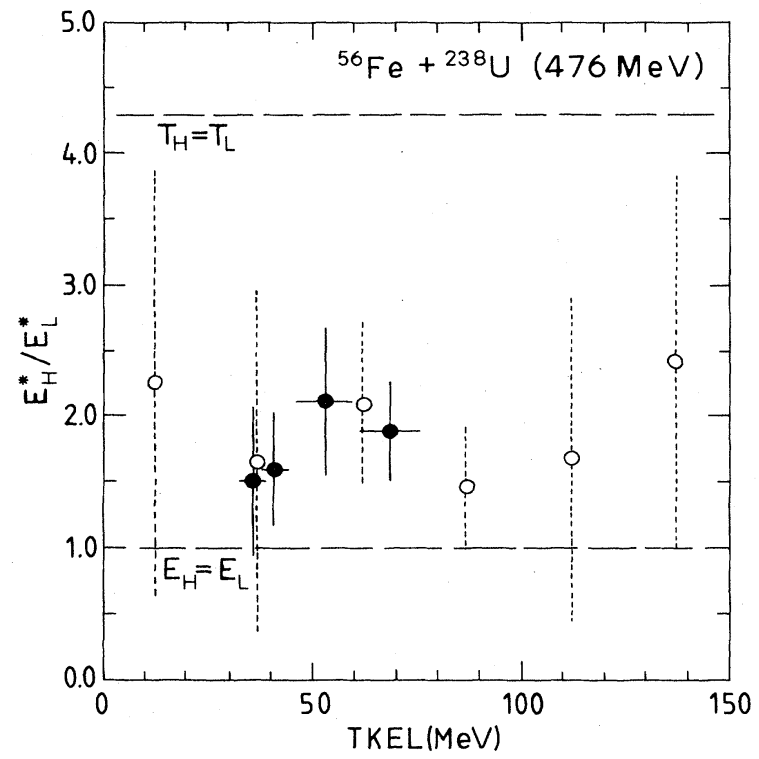

FIG. 2. Variation of the ratio of the excitation energy distributed between the heavy and light fragments with the energy loss for the reaction ${ }^{56} \mathrm{Fe}+{ }^{238} \mathrm{U}$ at $476 \mathrm{MeV}$. The values expected in the limits of equal division of the excitation energy and division leading to thermal equilibrium are shown by dashed horizontal lines. Full circles are the experimental results of Ref. 4 and empty circles are the results of the calculations of which estimated fluctuations are shown by vertical dotted lines.

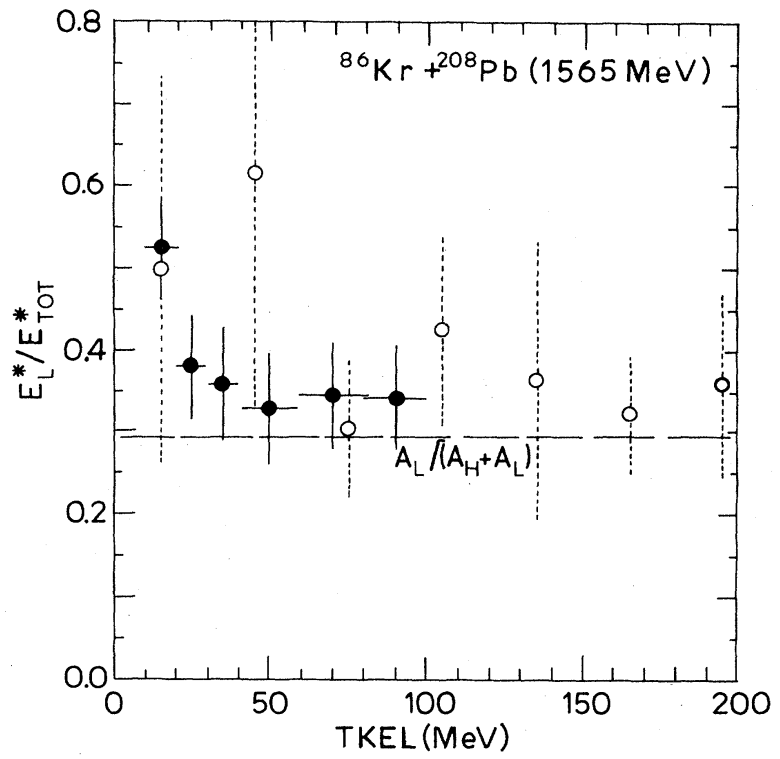

FIG. 3. Variation of the ratio of the light fragment excitation energy and the total one with the energy loss for the reaction ${ }^{86} \mathrm{Kr}+{ }^{208} \mathrm{~Pb}$ at $1565 \mathrm{MeV}$. The value expected in the limit of energy division according to the mass ratio is indicated by the horizontal dashed line. Full circles are the experimental data extracted from the results of Sohlbach et al. (Ref. 13) and the empty circles are the results of the calculations of which estimated fluctuations are shown by vertical dotted lines.

This work was supported in part by the Spanish Comision Asesora de Investigacion Cientifica y Tecnica, Contract No. 2868-83.

${ }^{1}$ D. Hilscher, J. R. Birkelund, A. D. Hoover, W. U. Schroeder, W. W. Wilcke, J. R. Huizenga, A. C. Mignerey, K. L. Wolf, H. F. Brever, and V. E. Viola Jr., Phys. Rev. C 20, 576 (1979).

${ }^{2}$ B. Tamain, R. Chechick, H. Fuches, F. Hanappe, M. Morjean, C. Ngo, J. Peter, M. Dakowski, B. Lucas, C. Mazur, M. Ribras, and C. Signarbieux, Nucl. Phys. A330, 253 (1979).

${ }^{3}$ Y. Eyal, A. Gavron, I. Tserruya, Z. Fraenkel, Y. Eisen, S. Wald, R. Bass, C. R. Gould, G. Kreyling, R. Renfordt, K. Stelzer, R. Zitmann, A. Gobbi, U. Lynen, H. Stelzer, I. Rode, and R. Bock, Phys. Rev. C 21, 1377 (1980).

${ }^{4}$ R. Vandenbosch, A. Lazzarini, D. Leach, D. K. Lock, A. Ray, and A. Seamster, Phys. Rev. Lett. 52, 1964 (1984).

${ }_{5}^{5}$ L. G. Moretto and E. G. Lanza, Nucl. Phys. A428, 173c (1984).

${ }^{6}$ J. Randrup, Nucl. Phys. A383, 468 (1982).

${ }^{7}$ K. T. R. Davies, K. R. S. Devi, S. E. Koonin, and M. R. Strayer, Heavy Ion Science, edited by D. A. Bromley (Plenum, New York, 1983).
${ }^{8}$ R. A. Broglia, C. H. Dasso, and A. Winther, in Nuclear Structure and Heavy Ion Collisions, Proceedings of the International School of Physics "Enrico Fermi," Course LXXVII, edited by R. A. Broglia, C. H. Dasso, and R. Ricci (North-Holland, Amsterdam, 1981), p. 327.

${ }^{9}$ R. A. Broglia, C. H. Dasso, G. Pollarolo, and A. Winther, Phys. Rev. Lett. 41, 25 (1978).

${ }^{10} \mathrm{~A}$. Bohr and B. Mottelson, Nuclear Structure (Benjamin, London, 1975), Vol. II.

${ }^{11}$ C. H. Dasso and G. Pollarolo (unpublished).

${ }^{12}$ R. A. Broglia, C. H. Dasso, and H. Esbensen, Prog. Part. Nucl. Phys. 4, 345 (1980); C. H. Dasso, in Nuclear Physics, edited by C. H. Dasso, R. A. Broglia, and A. Winther (North-Holland, Amsterdam, 1982), p. 565.

${ }^{13} \mathrm{H}$. Sohlbach, H. Freiesleben, P. Braun-Muzinger, W. F. W. Schneider, D. Schull, B. Kohlmeyer, M. Marinescu, and F. Puhlhofer, Phys. Lett. 153B, 386 (1985). 